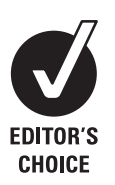

CHOICE

\title{
Self-paced exercise is less physically challenging than enforced constant pace exercise of the same intensity: influence of complex central metabolic control
}

\author{
P J Lander, ${ }^{1,2}$ R J Butterly, ${ }^{2}$ A M Edwards ${ }^{3}$
}

${ }^{1}$ UCOL Institute of Technology, Palmerston North, New Zealand:

${ }^{2}$ Leeds Metropolitan University, Carnegie Faculty of Sport \&

Education, Leeds, UK; ${ }^{3}$ Institute of Sport \& Exercise Science, James Cook University, Cairns, Australia

Correspondence to:

Dr A M Edwards, Institute of

Sport \& Exercise Science, James

Cook University, Cairns,

Queensland, Australia;

andrew.edwards@jcu.edu.au

Accepted 29 January 2009

Published Online First

5 February 2009

\section{ABSTRACT}

Objective: To examine whether self-pacing reduces the physiological challenge of performing $5000 \mathrm{~m}$ rowing ergometry exercise in comparison with a matchedintensity exercise condition in which a constant effort pacing strategy is enforced.

Methods: Nine healthy well-trained male participants volunteered to participate in three $5000 \mathrm{~m}$ rowing conditions (two submaximal and one maximal conditions) in an individualised order. In the submaximal conditions, participants were required to (1) perform $5000 \mathrm{~m}$ at a constant rating of perceived exertion (RPE 15-Hard) (SubRPE) or (2) perform $5000 \mathrm{~m}$ at an enforced constant pace equivalent to the mean power output (PO) of the SubRPE condition (SubEXT). A maximal condition (MaxTT) was included to disguise the purpose of the study and to facilitate an element of randomisation in the test sequence. Dynamic intratest responses were assessed every 30s: $\mathrm{PO}, \mathrm{VO}_{2}$, iEMG, core $\left(\mathrm{T}_{\mathrm{c}}\right)$ and skin temperatures $\left(T_{\mathrm{sk}}\right)$.

Results: There was no difference between performance times of the two submaximal trials. The mean PO represented 83.83 (SD 8.88)\% (SubRPE) and 83.40 (8.84)\% (SubEXT) of the mean MaxTT power output. $T_{C}$ (SubRPE:38.46 $(0.23)^{\circ} \mathrm{C}$, SubEXT:38.72 $(0.36)^{\circ} \mathrm{C}$; $p<0.01$ ), post-test BLa (SubRPE:5.24 (2.18), SubEXT:6.19 (2.51) mmol/l; $p<0.05)$ and iEMG $(p<0.05)$ were significantly elevated in SubEXT compared with SubRPE. There were no differences in the dynamics of $\mathrm{HR}$ or $\mathrm{VO}_{2}$ between SubEXT and SubRPE. The intratest stroketo-stroke variability of power output was significantly greater in the SubRPE condition compared with SubEXT $(p<0.01)$

Conclusions: Enforced constant paced exercise presents a significantly greater physiological challenge than selfpaced exercise. The ability to dynamically self-pace effort via manipulations of power output during exercise is an important behavioural response to homeostatic challenges and thus forms an integral part of a complex central regulatory process.

The ability to accurately self pace an exercise bout is an important feature of race and time trial performances. ${ }^{1-3}$ Self-paced exercise bouts are known to demonstrate considerable intratrial fluctuations of power output, ${ }^{4}$ and it is unlikely that this is simply due to random misjudgements of pace. It is probable that these fluctuations of power output are important behavioural responses during exercise at times when homeostasis is challenged. ${ }^{5}$ However, the importance of this observation requires researchers to consider the brain as a (central) feature of pacing and the development of fatigue.

Until recently, ${ }^{6-8}$ it had commonly been viewed that exercise of maximal intensity progressively induced a decrease in force production towards a terminal endpoint of fatigue at which the immediate cessation of exercise was a necessary consequence. ${ }^{9}$ This theory has often been used to attribute fatigue to impaired peripheral muscle contractile function, through either excessive accumulation of metabolic acidosis or the depletion of intramuscular fuels. ${ }^{10}$ However, such peripheral fatigue cannot easily explain all observations during endurance exercise, ${ }^{11}$ in particular those where performance improves in the end stages of a self-paced exercise bout. ${ }^{12-14}$

Several contemporary research studies have suggested that discreet alterations in pace are mediated through central neural control, by which muscle recruitment is manipulated as part of a regulatory process to maintain a reserve of motor units and thus avoid catastrophic fatigue. ${ }^{6-8}$ According to this central (governor) model, the regulation of exercise intensity (power output) is a behavioural response to both feedback information from peripheral receptors and feedforward (anticipatory) mechanisms which regulate exercise intensity to avoid the development of bodily harm. $^{311}$ Consequently, fluctuations in power output during exercise may be an important feature of a regulatory process, based on information from various peripheral systems (eg, muscle, respiratory, metabolic receptors) within a complex metabolic control system.

Previous work has shown biological variation to be an important feature in submaximal exercise. ${ }^{15}$ However, relatively few studies have thoroughly examined both the dynamic physiological and thermoregulatory responses to exercise in relation to the concept of pacing. ${ }^{12}{ }^{4}$ With the development of fast-response technologies, it is now feasible to examine the concept of pacing in more dynamic experimental conditions than was previously practical. For example, it is possible that thermoregulatory factors such as core and skin temperatures are dynamically related to the perception of effort during exercise through which alterations in pacing are linked to temperature regulation and/or muscle recruitment patterns. Nevertheless, there currently remains a lack of empirical data in which dynamic responses have been evaluated. 
We propose that the inter-relationship between conscious perceptions of effort (RPE) and subconscious metabolic control (mediating muscle recruitment) will result in physiologically meaningful non-random fluctuations of power output ${ }^{4}$ in selfpaced exercise, while enforced matched-intensity constant paced exercise will result in adverse physiological responses. The aim of this study was consequently to compare physiological responses to a $5000 \mathrm{~m}$ rowing exercise at a matched intensity in which the participants were (1) able to voluntarily fluctuate power output (self-paced) while performing exercise at a fixed rating of perceived exertion (RPE) or (2) were required to maintain a matched-intensity (enforced) constant power output.

\section{METHODOLOGY \\ Participants}

Nine healthy, well-trained male participants agreed to take part in this study (table 1). All were informed of the procedures in advance, and informed consent was provided prior to any data collection. The study was approved by the Central Regional Ethics Committee of New Zealand. All participants were recreational gymnasium users, and each received technical advice from a qualified rowing coach on using the rowing ergometer during a 2 -week familiarisation period.

\section{Preliminary testing}

At the beginning of the study, all participants performed a standardised familiarisation trial which consisted of a fourstage incremental protocol. The initial stage required participants to work for $4 \mathrm{~min}$ at RPE 11 (light), and each subsequent stage increased in intensity and decreased in time (3 min: RPE 13 (moderate), 2 min: RPE 15 (hard), 1 min: RPE 19 (very very hard)). This familiarisation protocol was subsequently used as a standardised priming exercise in each of the trials (fig 1).

Rowing ergometry was selected as a useful exercise modality for this study, as the power output attained (and effort) from each rowing stroke during the bout can be easily influenced by sensations of fatigue through up- and downregulation of effort when pulling the rowing handle. It is therefore highly sensitive to fluctuations in power during the test.

In all conditions, the air resistance of the ergometer flywheel was standardised by using the damper lever to apply a predetermined drag factor $130\left(10^{-6} \mathrm{Nms}^{2}\right) .{ }^{16}{ }^{17}$

On a separate and subsequent occasion all participants performed an incremental exercise test to volitional exhaustion on a Concept II rowing ergometer (Model D: Concept II, Tauranga, New Zealand) in our exercise laboratory for the determination of maximal aerobic power $\left(\mathrm{VO}_{2}\right.$ max). Oxygen uptake (Cortex MetaMax 3B, Cortex Biophysik, Leipzig, Germany) and power output (RowPro v2.006 software; Digital Rowing, Boston, Massachusetts) were continuously monitored stroke-to-stroke. Power output was visible via the Concept II display unit at all times.

\section{Experimental procedures}

Each subject completed three $5000 \mathrm{~m}$ rowing trials in three different experimental conditions in an individualised order. Condition 1 (Submaximal Self-paced using RPE scale; SubRPE) required the participants to complete $5000 \mathrm{~m}$ at a constant rating of perceived exertion (RPE: 15-Hard). Condition 2 (Submaximal Externally paced; SubEXT) required the participants to perform $5000 \mathrm{~m}$ at a constant power output equivalent to the mean power output attained in the SubRPE condition.
No visual feedback was provided in the SubRPE condition to ensure subjects self-paced, while only stroke-to-stroke power output was visible during the SubEXT condition, and participants received continual reinforcement to ensure the required power output was attained. A further experimental condition (Maximal Time Trial; MaxTT) was included to disguise the importance of the two submaximal conditions and to compare intensity of efforts, while also facilitating an element of randomisation in the test sequence. In the MaxTT condition, participants were instructed to perform $5000 \mathrm{~m}$ as fast as possible, while stroke-to-stroke power output was visible at all times (fig 1).

In the SubEXT condition, participants were deceived to believe that the required exercise intensity was based on a constant power output equivalent to that of ventilatory threshold attained in the baseline $\mathrm{VO}_{2}$ max test. This deception was conducted in an attempt to avoid participants realising that the two submaximal efforts in the three test series were matched for mean intensity and thus pacing the SubEXT trial on their previous SubRPE efforts.

The exercise trials were held at the same time of the day on each of the three occasions to avoid diurnal variations in body temperature, and were each separated by approximately 1 week. The participants were instructed to refrain from additional organised physical activity during the testing period and to maintain habitual exercise routines. The laboratory temperature was standardised at $18^{\circ} \mathrm{C}$ across all tests while relative humidity remained consistent (35-45\%). All participants consumed a beverage of water $2 \mathrm{~h}$ before the start of the test ( $5 \mathrm{ml}$ of water per kilogram body mass) to ensure comparable euhydration between participants and trials. ${ }^{18}$

\section{Oxygen uptake, heart rate and power output measurement}

Gas exchange and minute ventilation were continuously recorded breath by breath (Cortex MetaMax 3B, Cortex Biophysik, Leipzig, Germany). The system was calibrated before and verified after each test with standard calibration gases. Volume expired was measured by a volume measuring turbine, which was calibrated with a 31 syringe (Hans Rudolph, Kansas City, Missouri).

Whole blood capillary samples were drawn from the finger tip prior to exercise and at the immediate cessation of time-trial performances for the analysis of blood lactate concentration (Lactate Pro, Akray, Kyoto, Japan). Heart rates (HR) were continuously recorded (S610i, Polar, Kempele, Finland) throughout all exercise tests, time-aligned and averaged into 30 s intervals.

Stroke-to-stroke power output was assessed using the RowPro v2.006 software (Digital Rowing) in conjunction with the Concept II interface. After each trial, power output was time-aligned and averaged into $30 \mathrm{~s}$ intervals.

\section{Measurement of thermoregulatory factors}

Core temperature $\left(T_{c}\right)$ was measured via telemetry from the intestine using a silicon-coated thermometer pill (CorTemp2000, $\mathrm{HQ}$, Palmetto, Florida) which was swallowed by all participants $5 \mathrm{~h}$ before exercise to ensure that it would be past the stomach and insensible to swallowed hot or cold liquids. ${ }^{19}$ The calibration of the ingestible pills was checked prior to ingestion at four different temperatures against a certified mercury thermometer in a water bath at temperatures ranging from $30^{\circ} \mathrm{C}$ to $42^{\circ} \mathrm{C}$. In accordance with our earlier work, a linear regression equation was then used to adjust pill measurements. ${ }^{20}$ 
Table 1 Baseline anthropometric and cardiovascular characteristics of the participants

\begin{tabular}{|c|c|c|c|c|c|}
\hline & \multicolumn{5}{|c|}{ Anthropometric characteristics } \\
\hline & Height (m) & Weight (kg) & Age (years) & $\begin{array}{l}\mathrm{V}_{0_{2}} \text { peak } \\
(\mathrm{ml} / \mathrm{kg} / \mathrm{min})\end{array}$ & $\mathrm{HR}$ at $\mathrm{V}_{0_{2}}$ peak $(\mathrm{b} / \mathrm{min})$ \\
\hline Mean (SD) & $1.77(0.06)$ & $77.10(8.11)$ & $29(6)$ & $51.56(2.75)$ & $185.44(7.86)$ \\
\hline
\end{tabular}

Skin temperatures $\left(T_{\text {sk }}\right)$ were measured at four sites using stainless steel surface skin themistors (Grant Logistics, Cambridge). Temperatures were recorded continuously throughout the trial using a data logger (SQ400 Squirrel Data logger, Grant Logistics). The mean body skin temperature was calculated using the formula previously described by Ramanathan ${ }^{21}$ and others. ${ }^{22}{ }^{23}$ Measurements were taken of $\mathrm{T}_{\mathrm{c}}$ and $\mathrm{T}_{\mathrm{sk}}$ continuously throughout the trials in order to assess dynamic responses to exertion, time-aligned and then averaged into $30 \mathrm{~s}$ intervals for comparison with other dynamic exercise data sets.

\section{Measurement of surface iEMG}

Surface electrodes (Medi-Trace 230 Foam Electrode, Kendall Healthcare, Mansfield, Massachusetts) were placed $20 \mathrm{~mm}$ apart on the belly of the biceps brachii and vastus lateralis muscles, and a reference electrode was placed on the lateral aspect of the styloid process of the radius. ${ }^{24}{ }^{25}$ The skin surface was cleaned and shaved prior to electrode application in order to avoid interference and to increase adhesion; all electrodes were additionally fastened with medical adhesive tape. As rowing involves bilateral activation of the muscles, ${ }^{26}$ recordings were taken from only one side (right) of the body. Scores were not standardised against a standard isometric maximal voluntary contraction (MVC), as the dynamic nature of the movement pattern involved in rowing has been previously shown to elicit higher peak muscle sEMG in rowing than in the manoeuvre used to produce isolated maximal voluntary contractions. ${ }^{26}$ In addition, the plane of movement in rowing is difficult to replicate in MVC conditions, and pretrial evaluations did not support the use of that technique.

iEMG was recorded during the final $100 \mathrm{~m}$ of each $1000 \mathrm{~m}$ period using the Power Lab data acquisition system (Power Lab $\mathrm{AD}$ Instruments, NSW, Australia). Raw scores were digitally filtered (band pass filter; $20 \mathrm{~Hz}$ to $480 \mathrm{~Hz}$ ), digitised $(1 \mathrm{kHz}$ sampling rate) and stored (Chart 5 v5.5.5, $\mathrm{AD}$ Instruments, NSW, Australia). Each stroke was visually identified and quantified using the root mean square (rms) method, and the mean of three strokes at the end of each $1000 \mathrm{~m}$ interval across the three trials was then batched for the purposes of statistical comparisons.

\section{Data analysis}

Dynamic variations attributable to pacing were assessed by the measurement of oxygen uptake, heart rate and power output gained from the $30 \mathrm{~s}$ time-aligned data series of each outcome measurement.

A simple and effective means of determining time-domain variability is to calculate the standard deviation (SD) of each data point (ie, each $30 \mathrm{~s}$ time-aligned interval) as a series. Since variance is mathematically equal to the total power of spectral analysis, the SD of the data series reflects all the cyclic components responsible for variability in the period of recording, in this case the time trial. This method of analysis is frequently used in the study of heart rate variability. ${ }^{27} 28$ The standard deviation for each data series was therefore used to provide an overall comparative measure of dynamic time trial variability (ttv) between test conditions using the following outcome measurements: (1) oxygen uptake $\left(\mathrm{VO}_{2 \mathrm{ttv}}\right)$, (2) heart rate $\left(\mathrm{HR}_{\mathrm{ttv}}\right)$ and $(3)$ power output $\left(\mathrm{PO}_{\mathrm{ttv}}\right)$.

\section{Statistical analysis}

The statistical software package SPSS (version 11.0, SPSS, Chicago) was used for all statistical analysis. Parametric results were statistically compared using one-way repeated-measures analyses of variance (ANOVA) and post-hoc Tukey tests of

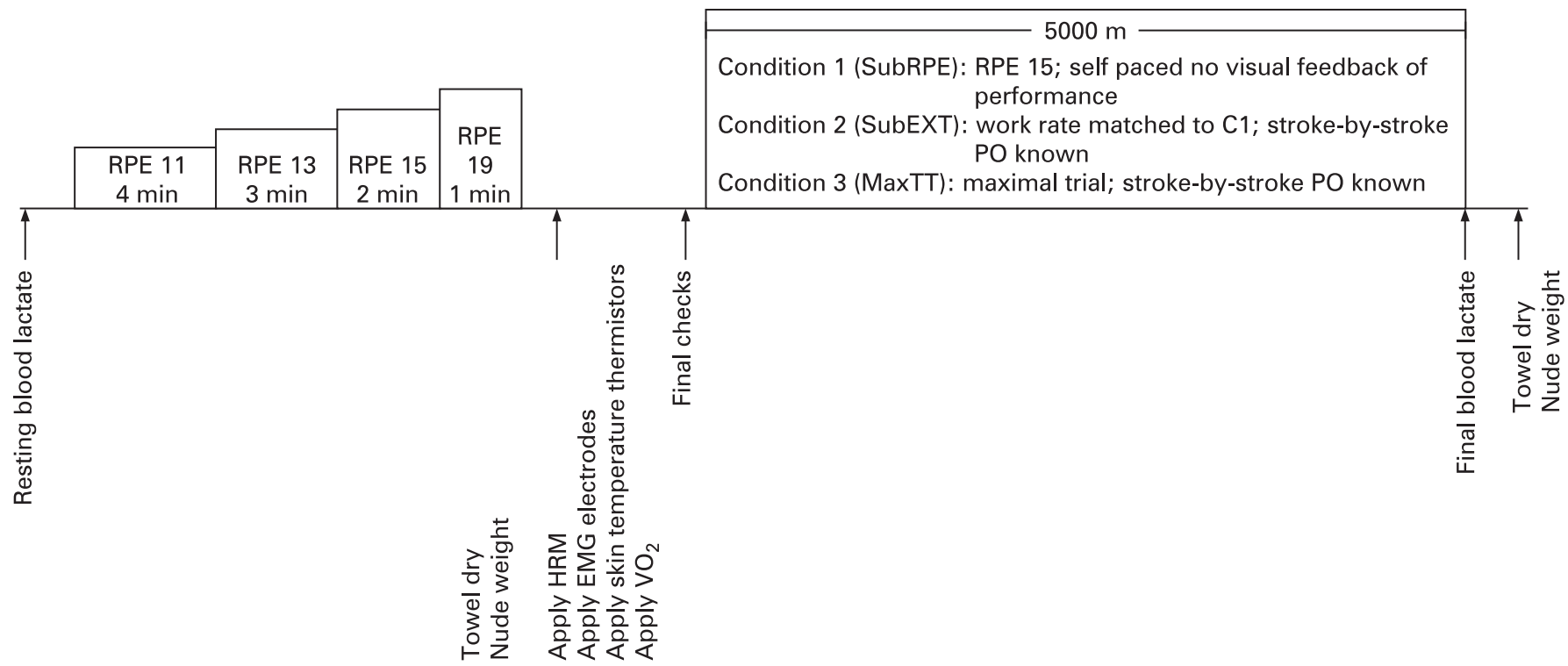

Figure 1 Experiment protocol for the three test sequence of rowing ergometer trials. 
Honest Significant Differences as appropriate. Other comparisons were made using paired Student t tests. Non-parametric data were assessed using the Friedman analysis of variance and MannWhitney $U$ tests. Probability values of less than 0.05 were considered significant. All results are expressed as mean (SD).

\section{RESULTS}

The fastest mean 5000 m performance time was observed in the MaxTT condition, and this was shorter in duration than both SubRPE $(p<0.01)$ and SubEXT $(p<0.01)$ (table 2$)$. There was no difference in the performance times of the two submaximal matched-intensity trials (SubRPE and SubEXT). The mean performance characteristics of $\mathrm{VO}_{2}, \mathrm{HR}$, and power output were not different between both submaximal conditions, but these were all significantly elevated in MaxTT (tables 2, 3).

There were no differences in mean or dynamics of oxygen uptake or heart rate between the submaximal conditions (table 3). However, the dynamics of power output $\left(\mathrm{PO}_{\mathrm{ttv}}\right)$ across the time-trials showed significantly greater variability in SubRPE compared with SubEXT $(\mathrm{p}<0.01)$ (table 2$)$. The variability of power output was further elevated in MaxTT condition compared with both the submaximal trials $(p<0.01)$ (table 2).

Mean $\mathrm{T}_{\text {sk }}$ was similar across all three (submaximal and maximal) conditions (fig 2). The mean $T_{c}$ was significantly lower in SubRPE than in both SubEXT $(p<0.05)$ and MaxTT $(p<0.01)$. There was no difference in mean $T_{c}$ between either SubEXT or MaxTT.

BLa concentrations taken immediately post-exercise were significantly elevated in SubEXT (6.2 (SD 2.5) mmol/l) compared with SubRPE $(5.2(2.2) \mathrm{mmol} / \mathrm{l})(\mathrm{p}<0.05)$. Both submaximal blood lactate responses were significantly lower when compared with the maximal trial $(10.9(2.4) \mathrm{mmol} / \mathrm{l})(\mathrm{p}<0.01)$.

Mean iEMG activity measured at both the vastus lateralis and biceps brachii was greater at each $1000 \mathrm{~m}$ interval in SubEXT when compared with SubRPE $(p<0.05)$. The mean iEMG activity of MaxTT was significantly higher than both the submaximal conditions at each $1000 \mathrm{~m}$ (fig 3).

Core temperature was not correlated with power output in any exercise condition, while $\mathrm{T}_{\text {sk }}$ was correlated with power output in both submaximal trials SubRPE $(r=0.67 ; p<0.01)$, SubEXT $(r=0.54, p<0.01)$ but not with MaxEXT. Immediate post-test evaluation of RPE in the SubEXT condition demonstrated a tendency for subjects to perceive that condition (RPE: 16 (1.9); $p=0.08$ ) to be more challenging than that of the prescribed RPE of 15 in the SubRPE condition. All subjects rated the MaxTT condition to be of maximal perceived effort (RPE: $20(0))$ on the RPE scale.

Scalar evaluation of $30 \mathrm{~s}$ power output data identified that all participants demonstrated a spurt of power (identified as a visible upward alteration in the trajectory of power output) at a

Table 2 Mean and dynamic responses of performance time and power output in the three experimental conditions

\begin{tabular}{|c|c|c|c|}
\hline & \multicolumn{3}{|c|}{ Performance outcome measurements } \\
\hline & \multirow{2}{*}{$\begin{array}{l}\text { Performance time } \\
\text { (s) }\end{array}$} & \multicolumn{2}{|l|}{ Power output } \\
\hline & & (W) & (ttv) \\
\hline SubRPE & $1300.11(77.53) \$$ & $162.16(26.63) \div$ & $17.85(3.60)+\%$ \\
\hline SubEXT & $1298.67(71.59) \ddagger$ & $161.33(26.51) \ddagger$ & $12.13(3.65)^{*}$ \\
\hline MaxTT & $1219.33(53.92)^{*} \dagger$ & $193.74(27.12)$ & $29.55(13.66)^{*}$ \\
\hline
\end{tabular}

Significantly different from Submaximal Self-paced using RPE scale $\mathrm{p}<0.01$;

${ }^{*}$ Submaximal Externally paced $\uparrow p<0.01$; Maximal Time Trial $p<0.01 \%$.

$\mathrm{ttv}$, Time trial variability.
Table 3 Mean and dynamic responses of oxygen uptake and heart rate in the three experimental conditions

\begin{tabular}{|c|c|c|c|c|}
\hline & \multicolumn{4}{|c|}{ Physiological (oxygen uptake and heart rate) responses } \\
\hline & \multicolumn{2}{|c|}{ Oxygen uptake } & \multicolumn{2}{|l|}{ Heart rate } \\
\hline & $(\mathrm{ml} / \mathrm{kg} / \mathrm{min})$ & (ttv) & (bpm) & (ttv) \\
\hline SubRPE & $36.87(4.96) \ddagger$ & $4.68(0.72) \%$ & $156.15(15.17)$ & $11.31(1.49)$ \\
\hline SubEXT & $35.88(5.62) \$$ & $4.30(0.82)$ & $153.06(17.42) \%$ & $12.62(1.56)$ \\
\hline MaxTT & $43.26(4.16)$ & $5.12(0.65)^{*}$ & $172.17(11.60)$ & $11.84(2.36)$ \\
\hline
\end{tabular}

Significantly different from Submaximal Self-paced using RPE scale $p<0.01$;

${ }^{*}$ Submaximal Externally paced $\uparrow p<0.01$; $†$ Maximal Time Trial $p<0.01$.

ttv, Time trial variability.

similar stage of their maximal trial (MaxTT) (89 (5)\% trial; range: $81-95 \%$ of trial duration).

\section{DISCUSSION}

The main finding from this study was that, in submaximal exercise, the enforced constant paced condition (SubEXT) posed significantly greater physiological and thermoregulatory challenges to homeostasis than the matched-intensity self-paced trial despite there being no difference in performance. Specifically, the SubEXT condition resulted in elevated mean core temperatures $(p<0.01)$, greater post-test blood lactate concentrations $(p<0.05)$ and elevated iEMG activity at both biceps brachii $(p<0.05)$ and vastus lateralis $(p<0.01)$. The most likely explanation for this appears to be that self-paced exercise facilitates the opportunity for individuals to continually modify effort via feedback and feedforward mechanisms in response to frequent homeostatic challenges. Thus, the greater time trial variability of power output $\left(\mathrm{PO}_{\mathrm{ttv}}\right)$ observed in the SubRPE condition compared with SubEXT $(p<0.01)$ may indicate the presence of a central regulatory mechanism.

The greater variation of $\mathrm{PO}_{\mathrm{ttv}}$ in the SubRPE condition compared with SubEXT was not accompanied by greater condition-specific variability in either $\mathrm{VO}_{2}$ or $\mathrm{HR}$. However, the similarity of $\mathrm{HR}$ and $\mathrm{VO}_{2}$ between SubRPE and SubEXT is logical, as power output is the variable manipulated as a behavioural response (to transient sensations of fatigue), and alterations in both $\mathrm{VO}_{2}$ and $\mathrm{HR}$ are therefore consequent with that behaviour, that is they are both responses to that change in power output. This delay in physiological response can also be explained via common system response times. For example, it is well known that the tau of oxygen uptake in response to dynamic changes in work is approximately 20-25 s among well trained participants, ${ }^{29}$ while the tau of heart rate is appreciably slower. ${ }^{30}$ Consequently, in self-paced exercise, dynamic variations in power output are probably too small and frequent for either $\mathrm{VO}_{2}$ or heart rate to discreetly follow each alteration. As noted by other authors, ${ }^{6}$ the importance of such dynamic responses have often been overlooked, probably due to the relatively recent emergence of fast-response technology. Nevertheless, such minor alterations in power output probably infer a mechanism by which voluntary behaviour (up- or downregulation of effort) maintains a constant metabolic challenge at a sustainable level throughout the bout. ${ }^{3}$ Behavioural change (pacing) therefore acts to defend homeostasis (e.g by defending core temperature and blood $\mathrm{pH}$ ) and this process is compromised where self-pacing is not facilitated.

The greater variability of power output observed in the maximal trial $(p<0.01)$ compared with the submaximal trials was a likely consequence of the greater freedom to alter pace in that condition in comparison with the restricted conditions (fixed RPE or fixed power output) of the submaximal trials. It is therefore 
Figure 2 Mean thermoregulatory responses to the matched intensity submaximal exercise trials (SubRPE, SubEXT) and the maximal condition (MaxTT).
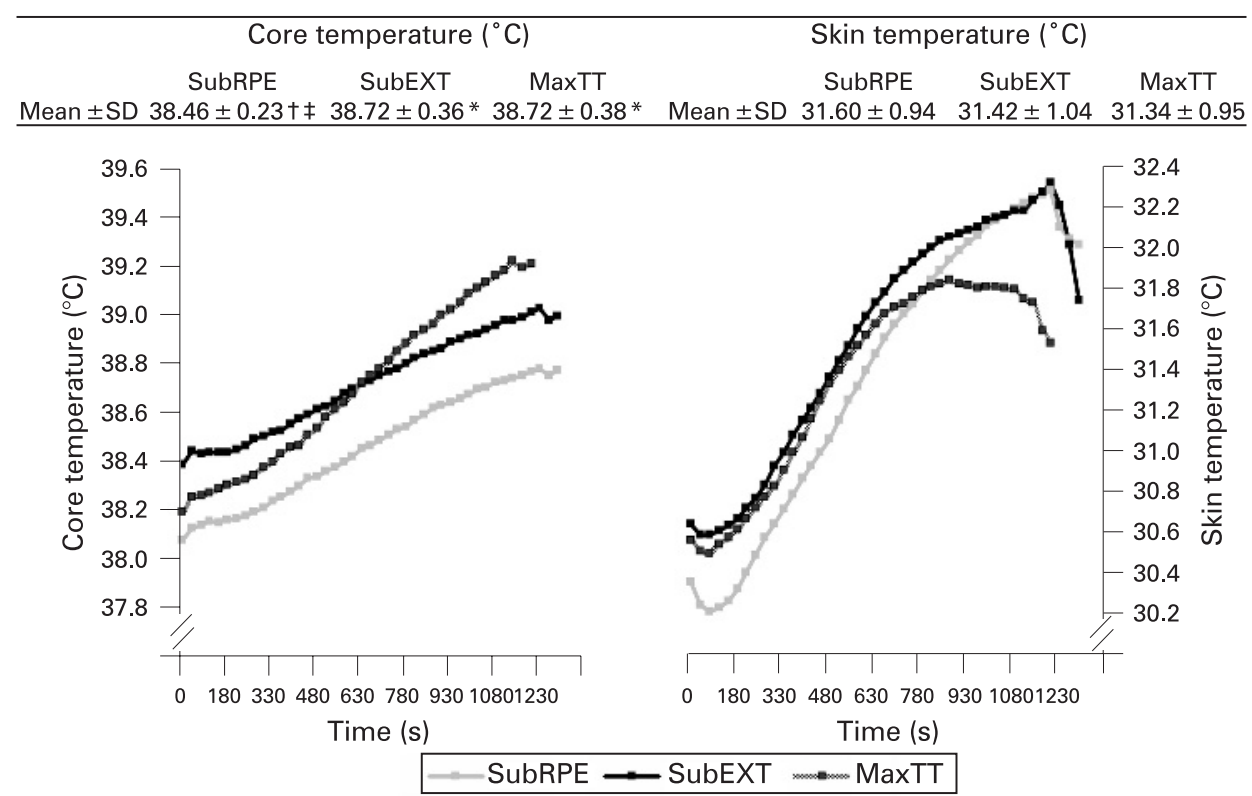

Significantly different from;

Submaximal self-paced using RPE scale $p<0.05^{*}$;

Submaximal externally paced $\dagger p<0.05+$;

Maximal time trial $\mathrm{p}<0.05 \ddagger$;

Submaximal self-paced using RPE scale $p<0.01 *$;

Submaximal externally paced $\dagger p<0.01 \dagger$;

Maximal time trial $p<0.01 \ddagger$

predictable that the maximal trial would demonstrate greater variability than the two submaximal conditions. Evaluation of the post-test RPE scores demonstrated that subjects tended to perceive the SubEXT condition (RPE: 16 (1).9) to be more challenging than the SubRPE condition (seven of the nine subjects rated it higher than SubRPE condition) but this did not reach statistical significance $(p=0.08)$. Two of the subjects did not rate the SubEXT condition to be more challenging than SubRPE although their metabolic responses (blood lactate concentrations and $T_{c}$ ) were elevated in the SubEXT condition. Our conclusion from this observation was that these two subjects were not able to verbally express their perceptions of effort as finely as their bodies were able to distinguish between the two submaximal conditions. The MaxTT condition produced a clear (and more obvious) distinction in RPE evaluation from the two submaximal conditions $(p<0.01)$ whereby all subjects rated their efforts as being at the top of the scale. The maximal trial was included in this study for several comparative purposes but most usefully to identify whether participants were able to distinguish between working at different levels of exertion in response to a $5000 \mathrm{~m}$ rowing exercise test. Participants in this experiment were clearly able to accomplish this task.

Previous studies ${ }^{12-14}$ have identified that an end spurt in performance tends to occur at $90 \%$ of task completion, and the maximal condition in this study was consistent with those observations. This appears to confirm that the increased final
Figure 3 Mean iEMG bar chart for each of three experimental conditions. BB, biceps brachii; VL, vastus lateralis.

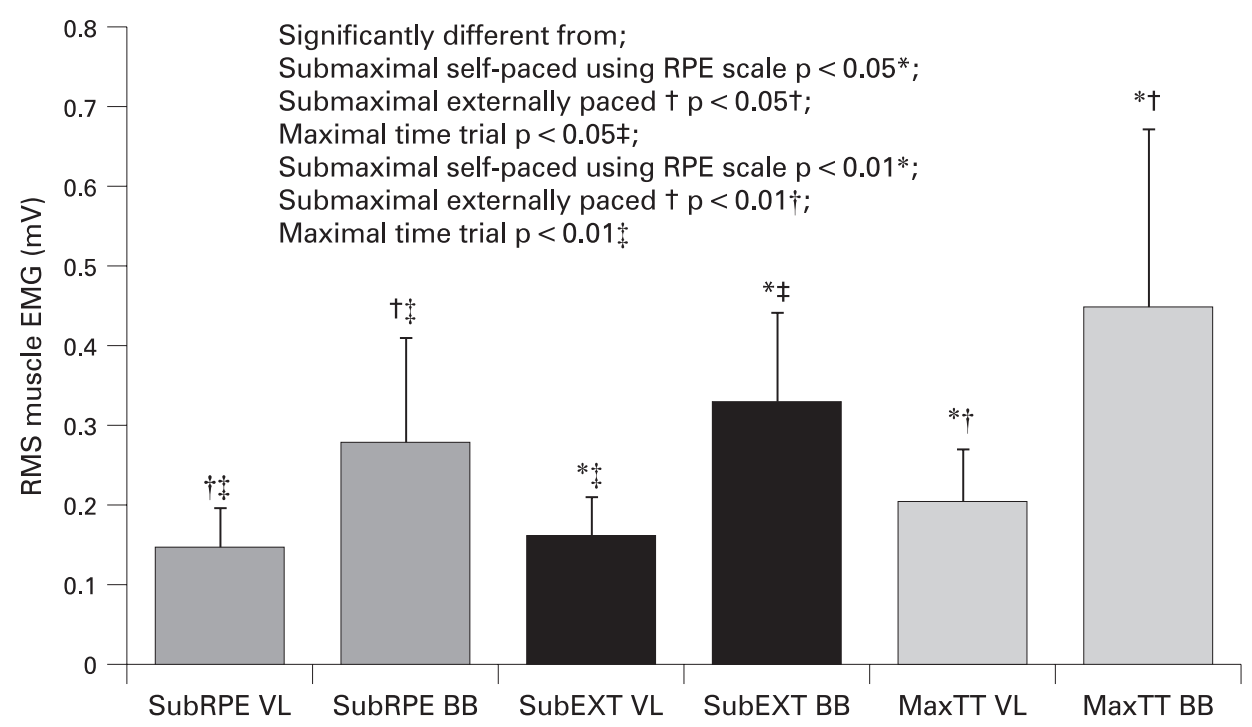




\section{What is already known on this topic}

- It is widely acknowledged that the ability to accurately self pace is an important feature of race and time trial performances.

- Non-random fluctuations of power output have recently been observed during self-paced exercise, which may be associated with system control mechanisms.

- A central (brain) governor model has been proposed to regulate both afferent and efferent physiological mechanisms to defend homeostasis via pacing during exercise and consequently avoid the development of bodily harm.

\section{What this study adds}

- This study demonstrates that self-pacing exercise poses a reduced metabolic challenge when compared with matchedintensity enforced constant paced submaximal exercise.

- The greater voluntary time trial variability of power output observed in a self-paced condition appears to be a behavioural response to metabolic disturbance, thereby resulting in greater up- and downregulation of effort with no net loss in performance compared with a matched intensity enforced constant paced submaximal condition.

- Use of modern fast-response technologies allows a higher capture rate of both dynamic physiological and thermoregulatory responses to exercise which are fundamental to the investigation of pacing during an exercise bout.

effort in maximal trials is representative of scalar rather than absolute pacing ${ }^{5}$ and provides little support for the concept that maximal intensity exercise progressively induces decreases in force production toward a terminal endpoint of fatigue.

No differences were seen in $T_{s k}$ across submaximal and maximal trials (fig 2), although the $T_{\text {sk }}$ of both SubRPE and SubEXT were correlated with power output (SubRPE: $r=0.67$, $p<0.01$; SubEXT: $r=0.54, p<0.01$ ). As the trajectories of $T_{s k}$ for both submaximal trials were similar (and also distinct from MaxTT), it is possible that $T_{\text {sk }}$ may have acted in some way as a regulatory mechanism in the submaximal conditions, but this was ineffectual for the maximal condition, whereby an optimal temperature (plateau) was reached at an earlier stage due to the greater physical cost of working in that condition. The most likely explanation for the similarity of skin temperatures between all conditions is probably that $T_{\text {sk }}$ progresses towards an optimal level during laboratory exercise and in the absence of further opportunities for convective cooling becomes ineffectual as a regulatory system when a steady state is attained. Further research needs to be conducted to confirm this observation.

It was anticipated that the MaxTT condition would result in significantly faster performances compared with the submaximal trials, but the similarity of physiological responses between SubEXT and MaxTT provides further evidence of the greater metabolic challenge of externally paced submaximal work in comparison with self-paced exercise. Indeed, where the ability to self pace is denied, the metabolic challenge progresses toward a similar level to that of maximal exercise.

\section{CONCLUSIONS}

This study demonstrates that self-pacing exercise poses a reduced metabolic challenge when compared with matchedintensity enforced constant paced submaximal exercise. It is likely that this is attributable to the ability to voluntarily fluctuate power output in accordance with transient sensations of fatigue during the exercise bout. The voluntary behavioural change to fluctuate pace is therefore a conscious decision based on subconscious physiological feedback from an array of peripheral receptors. Externally paced (enforced pacing) submaximal exercise thereby forces an individual to abandon their own pacing plan and minimises opportunities for self-managing the conscious signs of fatigue. This suggests that pacing is an important physiological mechanism to minimise the adverse conscious sensations of fatigue experienced during exercise which enables homeostasis to be defended during exercise.

To our knowledge, this study is the first to thoroughly examine both the cardiorespiratory and thermoregulatory responses to rowing performance in relation to matchedintensity self- and externally paced conditions. Further work is now required to establish whether this effect is consistent across more dynamic exercise challenges.

Competing interests: None.

Ethics approval: Ethics approval was provided by Central Regional Ethics Committe of New Zealand.

Patient consent: Obtained.

Provenance and peer review: Not commissioned; externally peer reviewed.

\section{REFERENCES}

1. Abbiss CR, Laursen PB. Describing and understanding pacing strategies during athletic competition. Sports Med 2008;38:239-52.

2. Foster C, Schrager M, Snyder A, et al. Pacing strategy and athletic performance. Sports Med 1994;17:77-85.

3. St Clair Gibson A, Noakes TD. Evidence for complex system integration and dynamic neural regulation of skeletal muscle recruitment during exercise in humans. Br J Sports Med 2004;38:797-806.

4. Tucker $\mathbf{R}$, Bester A, Lambert EV, et al. Non-random fluctuations in power output during self-paced exercise. Br J Sports Med 2006;40:912-17.

5. St Clair Gibson A, Lambert EV, Rauch LH, et al. The role of information processing between the brain and peripheral physiological systems in pacing and perception of effort. Sports Med 2006;36:705-22.

6. St Clair Gibson A, Schabort EJ, Noakes TD. Reduced neuromuscular activity and force generation during prolonged cycling. Am J Physiol Regul Integr Comp Physiol 2001;281:R187-96.

7. St Clair Gibson A, Lambert MI, Noakes TD. Neural control of force output during maximal and submaximal exercise. Sports Med 2001;31:637-50.

8. Noakes TD, St Clair Gibson A, Lambert EV. From catastrophe to complexity: a novel model of integrative central neural regulation of effort and fatigue during exercise in humans: summary and conclusions. Br J Sports Med 2005;39:120-4.

9. Hulleman M, De Koning JJ, Hettinga FJ, et al. The effect of extrinsic motivation on cycle time trial performance. Med Sci Sports Exerc 2007;39:709-15.

10. Kirkendall DT. Mechanisms of peripheral fatigue. Med Sci Sports Exerc 1990;22:444-9.

11. Lambert EV, St Clair Gibson A, Noakes TD. Complex systems model of fatigue: integrative homoeostatic control of peripheral physiological systems during exercise in humans. Br J Sports Med 2005;39:52-62.

12. Catalano JF. Effect of perceived proximity to end of task upon end-spurt. Percept Mot Skills 1973;36:363-77.

13. Catalano JF. End-spurt in addition of numbers. Percept Mot Skills 1974;39:121-2

14. Catalano JF. End-spurt following simple repetitive muscular movement. Percept Mot Skills 1974;39:763-6.

15. McLellan TM, Cheung SS, Jacobs I. Variability of time to exhaustion during submaximal exercise. Can J Appl Physiol 1995;20:39-51.

16. Smith B. Rowing New Zealand. In: Bishop B, Hume PA, eds. Guidelines for athlete assessment in New Zealand sport. Wellington: SESNZ, 2000.

17. Dudhia A. The physics of ergometers: measuring the damping. Available at: http:// www.atm.ox.ac.uk/rowing/physics/ergometer.html\#section7. (Accessed 7 May 2008).

18. Montain SJ, Coyle EF. Influence of graded dehydration on cardiovascular drift and hyperthermia during exercise. J App/ Physiol 1992;73:1340-50.

19. Sparling PB, Snow TK, Millard-Stafford ML. Monitoring core temperature during exercise: ingestible sensor vs. rectal thermistor. Aviat Space Environ Med 1993;64:760-3 
20. Edwards AM, Clark NA. Thermoregulatory observations in soccer match play: professional and recreational level applications using an intestinal pill system to measure core temperature. Br J Sports Med 2006;40:133-8.

21. Ramanathan NL. A new weighing system for mean surface temperature of the human body. J Appl Physiol 1964;19:531-3.

22. Marsh D, Sleivert G. Effect of precooling on high intensity cycling performance. Br J Sports Med 1999;33:393-7.

23. Mitchell D, Wyndham $\mathrm{CH}$. Comparison of weighting formulas for calculating mean skin temperature. J Appl Physiol 1969;26:616-22.

24. Basmajian JV, De Luca CJ. Muscles alive: their functions revealed by electromyography. 5th edn. Baltimore: Williams \& Wilkins, 1985.

25. De Luca CJ. Surface electromyography: detection and recording. Boston: Delsys, 2002.
26. Nowicky A, Burdett R, Horne S. The impact of ergometer design on hip and trunk muscle activity patterns in elite rowers:an electromyographic assessment. J Sports Sci Med 2005;4:18-28.

27. Achten J, Jeukendrup AE. Heart rate monitoring: applications and limitations. Sports Med 2003;33:517-38.

28. Aubert AE, Seps B, Beckers F. Heart rate variability in athletes. Sports Med 2003;33:889-919.

29. Edwards AM, Cooke CB. Oxygen uptake kinetics and maximal aerobic power are unaffected by inspiratory muscle training in healthy subjects where time to exhaustion is extended. Eur J App/ Physiol 2004;93:139-44.

30. Hughson RL. Exploring cardiorespiratory control mechanisms through gas exchange dynamics. Med Sci Sports Exerc 1990;22:72-9. 